\title{
A EFICIÊNCIA ECONÔMICO-FINANCEIRA DE OPERADORAS DE PLANOS DE SAÚDE: A INFLUÊNCIA DA MODALIDADE ${ }^{1}$
}

\author{
Daniele Oliveira Xavier ${ }^{2}$ \\ Antônio Artur de Souza
}

\begin{abstract}
RESUMO
Diante de um grande mercado que movimenta milhões anualmente e atende a mais de $20 \%$ da população brasileira quanto à saúde, fazem-se necessários estudos relacionados à eficiência econômico-financeira das Operadoras de Planos de Saúde (OPS). Soma-se a isso o fato que o mercado de saúde suplementar vem diminuindo ao longo dos últimos 15 anos. Dessa maneira, o estudo dessa eficiência mostra-se importante para a manutenção dessas empresas no mercado. Uma das formas de se analisar a situação financeira dessas organizações é por meio da utilização dos benchmarks de mercado. Para tanto, foram calculados alguns indicadores econômico-financeiros e realizados testes de Kruskal-Wallis e Análise Envoltória de Dados (DEA). Conclui-se que a modalidade a qual opera a OPS pode ser determinante para a sua eficiência e que alguns indicadores apresentam possibilidades de melhoria em grande parte das empresas, independente da modalidade.
\end{abstract}

Palavras-chave: Operadoras de planos de saúde. Eficiência econômico-financeira. Análise envoltória de dados (DEA).

\section{DETERMINANTS OF THE ECONOMIC AND FINANCIAL EFFICIENCY OF HEALTH PLAN OPERATORS: THE INFLUENCE OF THE MODALITY}

\begin{abstract}
Faced with a large market that moves billions of people and serves more than $20 \%$ of the Brazilian population regarding health, these studies may be related to the economic economy of the Health Plan Operators (OPS). This means that the supplemental healthcare market has been shrinking over the last 15 years. Thus, the study of this efficiency is important for the maintenance of these companies in the market. One way to analyze the financial situation in these situations is the average use of market benchmarks. For this, some economic and financial indicators were calculated and performed by Kruskal-Wallis and Data Envelopment Analysis (DEA). Conclude that a form of OPS quality operation can be decisive for its efficiency and that some indicators show possibilities for improvement in most companies, regardless of the modality.
\end{abstract}

\footnotetext{
${ }^{1}$ Como citar este artigo:

XAVIER, D. O.; SOUZA, A. A. de. A eficiência econômico-financeira de operadoras de planos de saúde: a influência da modalidade. ForScience, Formiga, v. 8, n. 2, e00707, jul./dez. 2020. DOI: 10.29069/forscience.2020v8n2.e707.
}

\footnotetext{
${ }^{2}$ Autor para correspondência: Daniele Oliveira Xavier, e-mail: danieleoliveiraxavier@gmail.com
} 
Keywords: Health plan operators. Economic and financial efficiency. Data envelopment analysis (DEA).

\section{INTRODUÇÃO}

O mercado privado de saúde brasileiro tem passado por diversas mudanças, das quais se destacam: perda de beneficiários, diminuição do número de empresas em operação, aumento dos custos assistenciais, dentre outras (ANS, 2018a). Os desafios para o setor de saúde suplementar são inúmeros. Araújo e Silva (2018) chamam atenção para o risco quanto à permanência dessas empresas no mercado mediante a tendência de aumento da sinistralidade verificada nessas organizações nos últimos anos. Uma das formas de se buscar a permanência dessas empresas no mercado seria a melhoria da sua eficiência, em especial a eficiência econômico-financeira. De acordo com Viacava et al. (2012, p. 924), “a eficiência em saúde é pensada como a relação entre o custo e o impacto dos serviços sobre a saúde da população, mantido um nível de qualidade determinado. Pereira e Silvestre (2009) chamam a atenção para a premissa de que é necessário um melhor controle da utilização de recursos pelo setor privado, pois se entende que faz parte de seus objetivos a obtenção de resultados financeiros que, eventualmente, podem levar ao racionamento no acesso aos procedimentos. Uma das maneiras de realizar esse controle é por meio da análise de desempenho. Souza et al. (2009) apontam para a importância da análise de desempenho da gestão financeira das diversas organizações prestadoras de serviços de saúde.

A avaliação de desempenho é um processo que permite realizar uma análise comparativa entre os resultados esperados e os resultados alcançados. Dentre as possibilidades de avaliação de desempenho comparativa, entre empresas, pode-se citar o benchmarking competitivo (DIMAGGIO; POWELL, 1983). Essa possibilidade fornece a comparação de uma entidade em relação às outras, apresentando, como resultado, a organização que detém as melhores práticas do mercado e que pode servir como exemplo a ser seguido pelas demais. De acordo com Macedo, Silva e Santos (2006), para identificar esse benchmark, uma das opções disponíveis é a utilização da técnica não-paramétrica conhecida como Análise Envoltória de Dados (DEA), que tem sido aplicada satisfatoriamente no setor financeiro.

Dados da Agência Nacional de Saúde (ANS, 2018b) demonstram que o setor tem mais de 47 milhões de usuários e teve receita bruta naquele ano da ordem de $\mathrm{R} \$ 118$ bilhões de reais. Apesar desses grandes números, é importante enfatizar que, atualmente, existem mais de 230 operadoras em processo de falência e em regime especial de liquidação. Dados da 
ANS (2018a) indicam que o crescimento do setor é expressivo desde os anos 80, tendo iniciado uma perda no número de beneficiários a partir do ano de 2015. Nishijima e Biasoto Junior (2006, p.2) afirmam que o mercado de saúde suplementar envolve vários tipos de desafios, principalmente quanto "às falhas de mercado decorrentes de assimetria de informação, tais como: problemas de seleção adversa, de agência e de risco moral, além dos problemas de externalidade dos bens de saúde". Dessa maneira, as ações e os serviços de saúde apresentam relevância pública, ainda que sua configuração institucional permita também a participação da iniciativa privada.

O atual mercado de saúde suplementar é composto por organizações das seguintes modalidades: (i) cooperativas médicas e odontológicas; (ii) medicina de grupo; (iii) filantropia; (iv) seguradoras especializadas em saúde; (v) autogestão; (vi) administradora de benefícios; e (vii) odontologia de grupo. O tipo de operadora que detém a maior quantidade de beneficiários é a cooperativa médica, seguida pelas medicinas de grupo. As cooperativas médicas são conhecidas principalmente pelas unidades Unimed, espalhadas pelo Brasil. Essas entidades são sociedades de pessoas sem fins lucrativos, constituídas conforme Lei 5.764, de 16 de dezembro de 1971. Já as medicinas de grupo são as organizações que operam planos e que não estão classificadas entre as demais modalidades.

Pires, Torres e Almeida (2013) informam que o setor das OPS apresenta grande acentuação na competição, sendo os grandes clientes empresariais o principal alvo dessa disputa. Pereira e Silvestre (2009) chamam a atenção para a redução no total de empresas que, no período entre 2005 e 2006, apresentou uma queda de 4,5\% no número de operadoras atuantes no Brasil (de 2.048 para 1.956 empresas) e aumento de 5,7\% no número de usuários (de 35,3 milhões para 37,3 milhões). Esse fato é corroborado pela baixa competição no mercado de saúde, no qual é possível observar que 30\% dos clientes concentram-se em apenas catorze operadoras, ou $1,1 \%$ do total de empresas em atividade no País, segundo dados da ANS de dezembro de 2006 (PEREIRA; SILVESTRE, 2009). De forma complementar, cabe citar que, no período entre 2006 e 2016, houve uma diminuição de aproximadamente $31 \%$ na quantidade de OPS com beneficiários no Brasil (ANS, 2017).

Diante das possibilidades de estudos e do contexto determinado, esta pesquisa orientase em torno do seguinte problema: A modalidade a qual as OPS fazem parte podem ter influência sobre a eficiência dessas entidades? O objetivo geral consiste em analisar a eficiência das OPS de modalidades diferentes com base em um conjunto de variáveis financeiras, à luz da teoria institucional. Sendo assim, o presente trabalho justifica-se pela necessidade de perceber a importância da diferenciação dos benchmarks de mercado 
formados em cada modalidade de operação. Tem-se como contribuição prática desse estudo a percepção de que as diferenças do sistema de regulação da saúde privada brasileira impostos às OPS tem efeitos também nos indicadores financeiros dessas organizações. A eficiência encontrada, bem como as possibilidades de melhoria, apresenta diferenciação a depender da modalidade de atuação.

\section{REFERENCIAL TEÓRICO}

\subsection{Isomorfismo e eficiência no setor de saúde}

De acordo com Damodaran (2007) pode-se afirmar que o coração das finanças pode ser representado pela avaliação que, em finanças corporativas, mostra-se como uma configuração ideal para aumentar o valor da empresa, alterando suas decisões de investimento, dividendos e financiamento. De acordo com Henry e Mark (2003), a avaliação representa uma mudança e/ou uma contribuição para os processos em curso que produzem consequências, sendo que essas consequências podem ser boas, ruins, neutras, mistas ou indeterminadas.

Damoradan (2007) afirma que a avaliação é a chave para muito do que é feito em finanças e que não se surpreende com a infinidade de abordagens de avaliação em uso. Altman (1968) afirma que uma tarefa da gestão empresarial extremamente importante, mas frequentemente muito difícil é a avaliação periódica e honesta da condição atual da empresa. De forma adicional, pode-se dizer também que as organizações devem medir o seu desempenho, a fim de verificar a sua posição no mercado (como um meio para estabelecer a sua posição, comparação de posição ou benchmarking, e monitorar o seu progresso), comunicar a sua posição (como um meio para comunicar o desempenho internamente e com o regulador), confirmar prioridades (como um meio para gerenciar o desempenho, custo e controle), e dar andamento de ações (como meio de motivação e recompensas) (ZSIDÓ, 2015). A identificação do posicionamento empresarial no mercado é de grande importância para a gestão empresarial, pois, por meio dele, é possível tomar decisões estratégicas que produzam impacto na gestão financeira. Ao fazer uma análise de mercado, é possível identificar as organizações que são benchmarks e que detêm as melhores práticas. Assim, para tal, deve-se ser capaz de identificar as organizações líderes em um mercado fazendo com que elas sejam vistas como uma referência a ser seguida pelas demais. A definição de uma organização como a detentora das melhoras práticas gerencias é conhecida como benchmark. 
A análise de benchmarks do mercado é realizada normalmente com base na eficiência de uma organização. De acordo com Zsidó (2015), uma visão geral da literatura nacional e internacional permite concluir que a maioria dos especialistas está definindo o desempenho dos negócios por meio de três fatores: eficiência, eficácia e eficiência econômica.

Melhorar a qualidade e a segurança dos cuidados de saúde é uma prioridade mundial (BRAITHWAITE et al., 2015). Allen et al. (2016) acreditam que a revisão das estruturas e processos formais das OPS é a chave para a prestação de serviços mais seguros, mais eficazes e mais eficientes. A revisão dessas estruturas pode ser vista como uma forma de se melhorar o desempenho dessas organizações e também como forma de manterem-se competitivas no mercado, ao optarem por aderirem a padrões já assumidos. Essa opção de aderir a padrões considerados como relevantes seja, por organizações do setor, do mercado em geral ou da academia, é considerada pela corrente institucionalista dentro da teoria organizacional como isomorfismo. Com base na teoria institucional espera-se que os indivíduos, organizações e grupos associem-se a padrões dominantes ou ditos adequados, quanto ao comportamento, normas, valores ou pressupostos (MACHADO-DA-SILVA; GONÇALVES, 2006). Dessa maneira, utiliza-se o termo isomorfismo para tratar, dentre outras possibilidades, da adoção de estruturas semelhantes em organizações diferentes (DIMAGGIO; POWELL, 1983). As organizações tendem a imitar outras organizações de sucesso quando elas enfrentam incerteza e ambiguidade no mercado e em suas práticas empresariais (DIMAGGIO; POWELL, 1983).

De acordo com Altman (1968), a detecção de funcionamento da empresa e de suas dificuldades é um assunto que tem sido particularmente suscetível à análise de relação financeira. Kaplan e Norton (2005) afirmam que medidas de desempenho financeiro podem indicar se a estratégia, a implementação e a execução da empresa estão a contribuir para a melhoria do negócio. Os autores enfatizam a importância de analisar os aspectos financeiros de uma organização e rebatem as afirmações de que as medidas financeiras são desnecessárias. Eles citam como argumentos a possibilidade de um sistema de controle financeiro bem projetado melhorar ao invés de inibir o programa de gestão da qualidade total de uma organização e a linha muito tênue e incerta sobre a suposta ligação entre a melhoria do desempenho operacional e sucesso financeiro.

De acordo com Ghalayini e Noble (1996), devido ao fato de as medidas de desempenho terem sido baseadas principalmente em sistemas de contabilidade, a maioria dessas medidas são centradas em dados financeiros (retorno do investimento, o retorno sobre as vendas, as variações de preços, as vendas por empregado, produtividade e lucro por 
unidade de produção). Assim, mostra-se a usabilidade dos indicadores financeiros para analisar o desempenho e eficiências das organizações.

\subsection{Estudos anteriores}

A avaliação de desempenho em organizações de saúde vem sendo objeto de diversos estudos nacionais e internacionais. Hollingsworth (2008) realizou uma meta-análise quanto à produção referente à medição da eficiência e produtividade da prestação de serviços de saúde por meio de uma revisão de 317 artigos internacionais publicados sobre a medição da fronteira de eficiência. De acordo com o autor, os estudos envolvendo eficiência e o setor de saúde passou a ser produzidos, em grande parte, a partir dos anos 2000. O autor concluiu que as técnicas mais utilizadas para esse tipo de análise são a análise envoltória de dados (nãoparamétrico) e a análise de fronteira estocástica (paramétrico).

Sampaio, Oliveira e Ignácio (2009) buscaram analisar e classificar 735 OPS no período entre 2001 e 2005 utilizando-se da ferramenta DEA e da lógica nebulosa. Utilizaramse, como inputs, ativo circulante, disponível e patrimônio líquido ou capital e, como output, contraprestações efetivas ou vendas. Os autores observaram que o porte das empresas influencia a eficiência em termos de controle do risco de (i) diferentes políticas de gestão; e (ii) insolvência.

Hashimoto (2010) analisou, em seu estudo, quatro modelos diferentes de eficiência das OPS no período de 2003 a 2008 utilizando a análise envoltória de dados (DEA) em todas as modalidades de operadoras. Como inputs e outputs do modelo, a autora utilizou os seguintes dados da contabilidade: taxa de cobertura; despesas médicas, de comercialização e administrativas; receitas; número de consultas por beneficiário e índice de não utilização. Como resultado desse estudo, observou-se que existem grandes diferenças entre modalidades e também entre regiões bem como indícios de que as modalidades tiveram diferentes reações à regulação.

Melo e Dantas (2018) chamam atenção para os possíveis impactos da junção da regulação da ANS com a promoção do direito à saúde por meio decisões judiciais em caráter liminar. Os autores enfatizam que é insustentável garantir o direito à saúde por meio de decisões judiciais mesmo no setor privado. Melo e Dantas (2018) afirmam ainda que a judicialização pode ter impacto no orçamento das operadoras e até mesmo promover o fechamento dela meio de uma intervenção da ANS quanto à sustentabilidade econômicofinanceiro dessas entidades. 
Avelar et al. (2019) apresenta o impacto da região de atuação delimitada pela ANS na distribuição dos resultados financeiros das OPS. Os autores concluíram que a região de localização normatizada pela Agencia Nacional foi a principal variável determinante para a distribuição de resultados de organizações classificadas como Medicina de Grupo.

Girdwood et al. (2019) consideram que a presença de um quadro regulamentar claro, em um ambiente de inovação, seria um catalisador para a mediação entre planos públicos e privados de saúde, de modo a garantir uma maior eficiência para o setor e oferecer serviços de menor custo à população.

Com base no exposto, percebe-se que o campo tem abordado o tema de avaliação de desempenho econômico-financeiro em sua grande parte utilizando dados brutos das demonstrações contábeis e o modelo DEA para determinar o desempenho. Percebe-se a necessidade de estudos que utilizem indicadores financeiros para avaliar o desempenho econômico-financeiro e que proponham um modelo de gestão baseado em benchmarks do mercado.

\section{MATERIAL E MÉTODOS}

\subsection{Caracterização do estudo}

A pesquisa desenvolvida pode ser classificada como descritiva e ex post facto, com abordagem quantitativa de dados, segundo autores como Lakatos e Marconi (2004). Creswell (2007) indica que na pesquisa quantitativa, o pesquisador deve testar uma teoria ao especificar as hipóteses a partir de dados numéricos e procedimentos estatísticos e matemáticos. Os dados utilizados para o desenvolvimento da pesquisa são secundários, ou seja, contêm pelo menos um nível de interpretação inserido entre o fato e o seu registro (COOPER; SCHINDLER, 2003). Os dados, do período de 2010 a 2015, foram coletados no site da ANS, sendo selecionados a partir da adoção plena das normas do International Financial Reporting Standards (IFRS).

Cabe explicar que o universo ou população de uma pesquisa corresponde ao "[...] conjunto de todos os casos que concordam com determinadas especificações" (SAMPIERI; COLLADO; LUCIO, 2006, p. 253). A amostra foi composta por operadoras do tipo médicohospitalares, pois, em 2015, essas organizações representavam $72 \%$ das operadoras de planos de saúde suplementar (ANS, 2018a). A quantidade de organizações a serem analisadas em cada segmento (amostra) foi determinada a partir da amostragem probabilística de uma 
população finita. Para tanto, utilizou-se a fórmula proposta por Stevenson (2001) que determina o tamanho amostral probabilístico quando o número de elementos que compõe a população é determinado e conhecido. Desse modo, essa pesquisa analisou ao todo 498 organizações diferentes que, não necessariamente, estavam presentes em todos os anos do período analisado.

Para o presente estudo, optou-se por analisar as seguintes modalidades: Autogestão, Cooperativa Médica, Filantropia e Medicina de Grupo. As cooperativas médicas classificamse como organizações de pessoas sem fins lucrativos. As entidades de autogestão têm como mantenedor grandes empregadores que gerenciam planos de saúde próprios para seus funcionários e dependentes mediante o credenciamento de hospitais e médicos. As entidades de filantropia são definidas como lucrativos e deve ter o certificado de entidade beneficente de assistência social. Por fim, as organizações conhecidas com medicina de grupo aquelas que os planos de saúde cujas características não se enquadram nas outras definições. Optou-se ainda por excluir as seguintes modalidades nesse estudo: seguradoras especializadas em saúde; administradoras de benefícios, odontologia de grupo e cooperativa odontológica. A exclusão se deve ao fato dessas entidades não assumirem a maioria dos riscos relacionados às operações de saúde, que são inerentes às demais modalidades.

\subsection{Formulações dos modelos}

Para Golany e Roll (1989), as análises de eficiência são ferramentas vitais de controle gerencial para avaliar o grau em que os insumos são utilizados no processo de obtenção dos produtos desejados. Uma das formas de se analisar essa eficiência é utilizando o modelo de Análise de Envoltória de Dados (Data EnvelopmentAnalysis - DEA). Mello et al. (2005, p.1) definem DEA como "ferramenta matemática para a medida de eficiência de unidades produtivas". Assim, o modelo DEA tem como objetivo "identificar as unidades de produção DMUs (por exemplo, organizações) eficientes, medir e localizar a ineficiência e estimar uma função de produção linear por partes, que fornece o benchmark (referência) para as DMUs ineficientes" (MELLO et al., 2005, p.16). Para tanto, foi necessário definir os indicadores que seriam utilizados no modelo. Para a composição dos modelos foram selecionados apenas indicadores que não apresentaram uma forte correlação de Spearman. Optou-se pela utilização do teste de Spearman pela característica não paramétrica do teste.

Para o cálculo da eficiência foi utilizado o modelo DEA, modelo BCC orientado para o produto. O modelo BCC (dos autores, Banker, Charnes e Cooper) pressupõe que as DMU's 
avaliadas apresentam retornos variáveis de escala, podendo ser crescentes ou decrescentes na fronteira eficiente. Com o intuito de analisar diversos aspectos organizacionais e fornecer informações sobre grande parte da análise financeira, propõe-se utilizar dois tipos de modelos diferentes.

Os escores de eficiência foram considerados de duas formas: (i) modelo 1 - escores obtidos ao se analisar um mesmo grupo de organizações; (ii) modelo 2 - escores obtidos ao analisar todas as organizações em conjunto - independentemente de a que grupo/modalidade de organizações elas pertençam. Em cada ano, cada um dos modelos foi analisado com o modelo DEA BCC orientado ao output. Os modelos DEA foram calculados a partir de dois programas, a saber: Software R para Windows na versão 3.3.2 e o Software SIAD (Sistema Integrado de Apoio à Decisão), desenvolvido por Ângulo Meza, Biondini Neto e Ribeiro (2005). Foi ainda analisada a eficiência separada por grupo/modalidade e conjuntamente, sem discriminação de grupo. O desing dos modelos utilizados está apresentado no Quadro 1.

\begin{tabular}{|c|c|c|c|c|}
\hline Inputs & Outputs & Orientação & Modelos & Amostra \\
\hline ENDIV & ROA & \multirow[t]{5}{*}{ Output } & \multirow[t]{3}{*}{1} & \multirow{3}{*}{$\begin{array}{l}\text { Modalidades de OPS analisadas } \\
\text { separadamente }\end{array}$} \\
\hline $\mathrm{CE}$ & ROE & & & \\
\hline DM & MEBITDA & & & \\
\hline COMBA & LG & & \multirow[t]{2}{*}{2} & \multirow{2}{*}{$\begin{array}{l}\text { Modalidades de OPS analisadas } \\
\text { conjuntamente }\end{array}$} \\
\hline PMCR & & & & \\
\hline
\end{tabular}

Quadro 1 - Modelos de análise da eficiência utilizados - DEA BCC Fonte: Autores (2018).

Assim, optou-se por analisar os indicadores tidos como tradicionais para se analisar o desempenho das OPS. No Quadro 2 são apresentadas as fórmulas para cada um dos indicadores econômico-financeiros utilizados, com base na descrição da ANS (2018a).

Posteriormente, para identificar outras características que não constam no modelo DEA BCC e que podem estar relacionadas com a eficiência das OPS, foram realizados novos testes com os escores de eficiência. Nessa etapa foi empregada ainda a técnicas de análise Kruskal-Wallis. Essa técnica testa, de forma não paramétrica, se duas ou mais amostras provêm de populações semelhantes ou de populações diferentes (MARÔCO, 2010). 
XAVIER, D. O.; SOUZA, A. A. de. A eficiência econômico-financeira de operadoras de planos de saúde: a influência da modalidade

\begin{tabular}{|c|c|c|c|}
\hline Nome & Sigla & Fórmula & Descrição \\
\hline $\begin{array}{l}\text { Endividamen } \\
\text { to }\end{array}$ & ENDIV & $\frac{\text { Passivo circulante + Exigivel a longo prazc }}{\text { Ativo total }}$ & $\begin{array}{l}\text { Mostra a } \\
\text { relação entre o } \\
\text { Exigível total e } \\
\text { o Ativo total. }\end{array}$ \\
\hline $\begin{array}{l}\text { Composição } \\
\text { do } \\
\text { endividament } \\
\text { o }\end{array}$ & $\mathrm{CE}$ & $\frac{\text { Passivo circulante }}{\text { Exigivel total }}$ & $\begin{array}{l}\text { Indica quanto } \\
\text { da dívida total } \\
\text { vence no curto } \\
\text { prazo }\end{array}$ \\
\hline $\begin{array}{l}\text { Índice de } \\
\text { despesas } \\
\text { assistenciais, } \\
\text { ou despesas } \\
\text { médicas, ou } \\
\text { ainda, } \\
\text { sinistralidade }\end{array}$ & $\mathrm{DM}$ & $\frac{\text { Eventos indenizáveis liquidos }}{\text { Contraprestações efetivas }}$ & $\begin{array}{l}\text { Mostra a } \\
\text { relação entre } \\
\text { despesas } \\
\text { assistenciais e o } \\
\text { total das } \\
\text { receitas com } \\
\text { operação de } \\
\text { planos de saúde }\end{array}$ \\
\hline $\begin{array}{l}\text { Índice } \\
\text { combinado } \\
\text { ampliado }\end{array}$ & COMBA & $\frac{\text { Desp.adm.+Desp.de comerc }+ \text { Eventos inden.líquido }}{\text { Contraprestações Efetivas + Resultado financeiro líquido }}$ & $\begin{array}{l}\text { Mostra a } \\
\text { relação entre } \\
\text { despesas } \\
\text { operacionais e } \\
\text { as receitas }\end{array}$ \\
\hline $\begin{array}{l}\text { Prazo médio } \\
\text { de } \\
\text { contraprestaç } \\
\text { ões a receber }\end{array}$ & PMCR & {$\left[\frac{\text { Créditos operaçōes de saúde }}{\text { Contraprestações efetivas }}\right] x 360$} & $\begin{array}{l}\text { Indica o tempo } \\
\text { médio que a } \\
\text { operadora leva } \\
\text { para receber as } \\
\text { vendas } \\
\text { realizadas }\end{array}$ \\
\hline $\begin{array}{l}\text { Taxa de } \\
\text { retorno sobre } \\
\text { o ativo total }\end{array}$ & ROA & $\frac{\text { Resultado liquido }}{\text { Ativo total }}$ & $\begin{array}{l}\text { Mostra a } \\
\text { relação entre o } \\
\text { resultado } \\
\text { líquido e o ativo } \\
\text { total. }\end{array}$ \\
\hline $\begin{array}{l}\text { Taxa de } \\
\text { retorno sobre } \\
\text { o patrimônio } \\
\text { líquido }\end{array}$ & ROE & $\frac{\text { Resultado liquido }}{\text { Patrimônio líquido }}$ & $\begin{array}{l}\text { Indica a relação } \\
\text { entre o } \\
\text { resultado } \\
\text { líquido e o } \\
\text { patrimônio } \\
\text { líquido }\end{array}$ \\
\hline $\begin{array}{l}\text { Margem } \\
\text { EBITDA }\end{array}$ & MEBITDA & $\frac{\text { Result. Líq + DespFinanc +IR + CSLL - ImpDifer +Deprec + Amort }}{\text { Contraprestações efetivas }}$ & $\begin{array}{l}\text { Mostra o lucro } \\
\text { antes de Juros, } \\
\text { Impostos, } \\
\text { Depreciação e } \\
\text { Amortizção }\end{array}$ \\
\hline $\begin{array}{l}\text { Liquidez } \\
\text { geral }\end{array}$ & LG & $\begin{array}{l}\text { Ativo circulante + Realizável a longo prazo } \\
\text { Passivo circulante + Exigível a longo prazo }\end{array}$ & $\begin{array}{l}\text { Mostra a } \\
\text { capacidade de } \\
\text { pagamento no } \\
\text { longo prazo }\end{array}$ \\
\hline
\end{tabular}

Quadro 2 - Fórmulas dos indicadores econômico-financeiros Fonte: ANS (2018).

\section{RESULTADOS E DISCUSSÃO}

Buscando identificar os indicadores financeiros e características organizacionais mais bem relacionadas à eficiência das OPS da amostra analisada, realizou-se, inicialmente, o teste de Kolmogorov-Sminorv para verificar se a distribuição das variáveis métricas era similar à distribuição normal. Após confirmar que a grande maioria das variáveis propostas não apresentava distribuição similar à normal, procedeu-se ao cálculo da eficiência econômico- 
financeira das organizações da amostra a partir dos modelos 1 e 2 propostos para esta pesquisa. A análise da eficiência para o presente estudo foi realizada por meio de dois modelos diferentes, descritos no quadro 1. A fim de se ter uma visão mais ampla dos resultados obtidos em cada ano e modelo, optou-se por apresentar a eficiência média das organizações analisadas. A evolução da eficiência média ao longo dos anos é apresentada por meio da Figura 1 e da Tabela 1.

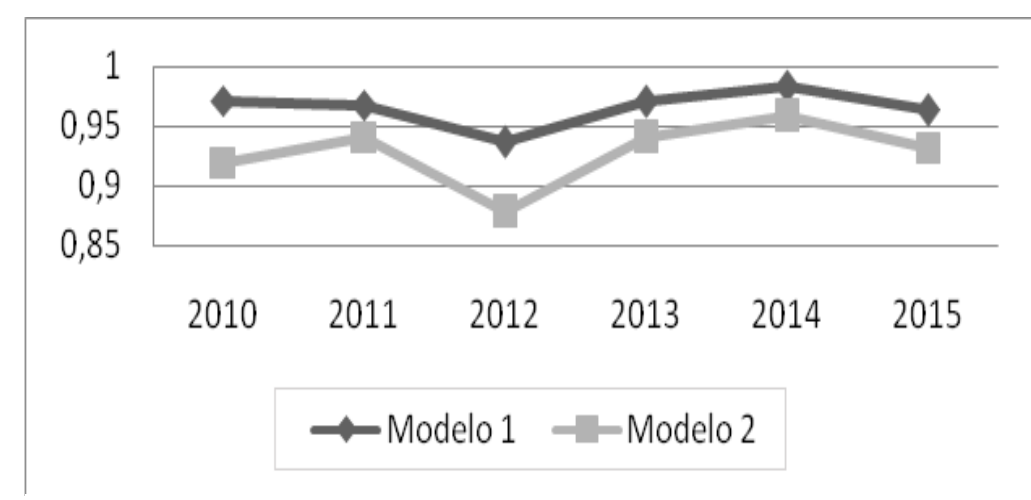

Figura 1 - Eficiência média das OPS analisadas Fonte: Dados da pesquisa.

Tabela 1 - Estatísticas Descritivas - Eficiência das OPS analisadas

\begin{tabular}{l|l|l|l|l|l|l|l}
\hline & & $\mathbf{2 0 1 0}$ & $\mathbf{2 0 1 1}$ & $\mathbf{2 0 1 2}$ & $\mathbf{2 0 1 3}$ & $\mathbf{2 0 1 4}$ & $\mathbf{2 0 1 5}$ \\
\hline \multirow{4}{*}{ Modelo 1 } & Média & $97 \%$ & $97 \%$ & $94 \%$ & $97 \%$ & $98 \%$ & $96 \%$ \\
\cline { 2 - 8 } & Mínimo & $78 \%$ & $83 \%$ & $47 \%$ & $83 \%$ & $86 \%$ & $82 \%$ \\
\cline { 2 - 8 } & Máximo & $100 \%$ & $100 \%$ & $100 \%$ & $100 \%$ & $100 \%$ & $100 \%$ \\
\cline { 2 - 8 } & Desvio-padrão & $3 \%$ & $3 \%$ & $6 \%$ & $2 \%$ & $2 \%$ & $3 \%$ \\
\hline \multirow{5}{*}{ Modelo 2 } & Média & $92 \%$ & $94 \%$ & $88 \%$ & $94 \%$ & $96 \%$ & $93 \%$ \\
\cline { 2 - 8 } & Mínimo & $78 \%$ & $75 \%$ & $47 \%$ & $78 \%$ & $75 \%$ & $76 \%$ \\
\cline { 2 - 8 } & Máximo & $100 \%$ & $100 \%$ & $100 \%$ & $100 \%$ & $100 \%$ & $100 \%$ \\
\cline { 2 - 8 } & Desvio-Padrão & $4 \%$ & $3 \%$ & $5 \%$ & $3 \%$ & $2 \%$ & $4 \%$ \\
\hline
\end{tabular}

Fonte: Dados da pesquisa.

O modelo 1 analisou a eficiência da amostra para cada tipo de modalidade, das OPS, comparadas separadamente. Ou seja, no modelo 1, organizações que são da modalidade Medicina de Grupo foram comparadas apenas com organizações do mesmo segmento. Já o modelo 2, analisou a eficiência da mesma amostra em conjunto, ou seja, desconsiderando a modalidade da qual a OPS faz parte. A partir dos resultados encontrados para os escores de eficiências dos modelos 1 e 2, pode-se perceber que a eficiência do setor se apresenta de forma homogênea. Em todos os seis anos analisados, a eficiência média não foi menor que $88 \%$. Essa homogeneidade pode ser um reflexo do isomorfismo previsto pela teoria institucional em ambientes de risco e altamente regulado. Hung (2008) afirma que o 
isomorfismo é uma tentativa por parte das empresas de limitar a sua incerteza modelando uma atividade seguindo um exemplo bem-sucedido. Assim, em cenários de grandes riscos, altamente competitivos e regulados a variabilidade de resultados/produtos é limitada, pois o isomorfismo surge como uma reação que pretende blindar os negócios empresariais.

$\mathrm{Na}$ Tabela 1, percebeu-se ainda que as eficiências do modelo 1 e modelo 2 foram diferentes, apesar de seguirem uma mesma tendência ao longo dos anos. A eficiência do modelo 1 foi maior do que os escores do modelo 2. Assim, percebe-se que, quando as organizações são comparadas entre seus pares, elas obtêm eficiência maior do que quando são comparadas com todo o setor, independentemente da modalidade. Dessa maneira, foi possível ter indícios de que as modalidades nas quais as organizações se encontram registradas têm influência sobre sua eficiência.

Matematicamente, é possível se observar uma variação ao se analisar os resultados da eficiência do modelo 1 e 2 . Ou seja, é possível se identificar que ao se analisar as operadoras com seus pares ou em conjunto, têm-se resultados diferentes. Assim, é possível concluir que fazer parte de uma das modalidades pode acarretar uma diferença na eficiência da organização. Entretanto, ao analisar a Figura 1, é possível identificar uma tendência de resultado do setor, para o modelo 1 e para o modelo 2. De forma análoga, ao se analisar os desvios-padrões dos modelos 1 e 2, da Tabela 1, é possível identificar uma certa paridade entre os modelos. A variabilidade entre as eficiências da amostra é pequena. Dessa maneira, encontram-se indícios de que as modalidades possam fazer diferença na eficiência das OPS, mas que de forma geral, essas organizações estão inseridas em um mesmo contexto, e consequentemente, estão suscetíveis a problemas similares. Malta et al. (2004) explica que a regulação pública realizada pela ANS atinge todas as OPS, nas seguintes áreas: saúde financeira, direito dos consumidores e questões relacionadas a ofertas de produtos.

A Tabela 2 apresenta a distribuição das OPS consideradas eficientes pelo modelo separadas por modalidade e por ano. A amostra das organizações de Autogestão estava em torno de 100 empresas, sendo que, a partir do modelo analisado, não mais que $25 \%$ delas foram consideradas eficientes. Isso demonstra uma variabilidade entre os indicadores do grupo Autogestão. Essa variabilidade pode indicar uma maior liberdade na tomada de decisão administrativa e também um maior espaço para inovações. No entanto, a maioria das organizações dessa modalidade não se encontra na fronteira da eficiência, indicando assim uma possível necessidade/possibilidade de melhoria por meio de seus benchmarks de mercado. 
XAVIER, D. O.; SOUZA, A. A. de. A eficiência econômico-financeira de operadoras de planos de saúde: a

Tabela 2 - Operadoras consideradas eficientes por modalidade e por ano - Modelo 1

\begin{tabular}{l|c|c|c|c|c|c}
\hline Modalidade & $\mathbf{2 0 1 0}$ & $\mathbf{2 0 1 1}$ & $\mathbf{2 0 1 2}$ & $\mathbf{2 0 1 3}$ & $\mathbf{2 0 1 4}$ & $\mathbf{2 0 1 5}$ \\
\hline Autogestão & 20 & 21 & 16 & 9 & 16 & 24 \\
\hline Cooperativa & 28 & 21 & 17 & 12 & 17 & 17 \\
\hline Filantropia & 24 & 20 & 22 & 20 & 22 & 16 \\
\hline Medicina de Grupo & 31 & 18 & 12 & 15 & 22 & 24 \\
\hline Total de OPS eficientes & 103 & 80 & 67 & 56 & 77 & 81 \\
\hline Total de OPS analisadas & 452 & 443 & 445 & 446 & 450 & 447 \\
\hline
\end{tabular}

Fonte: Dados da pesquisa.

Para as organizações do tipo Cooperativas e Medicina de Grupo, a amostra analisada nessa pesquisa foi composta, no mínimo por 135 OPS, por ano. A partir da Tabela 2 percebese que as OPS eficientes encontradas, em 3 dos anos analisados, representou menos de $15 \%$ da amostra. Essas oscilações das organizações eficientes ao longo dos anos podem representar (i) uma mudança nas estratégias empresariais, (ii) uma necessidade de atendimento a uma norma legal imposta pelos órgãos reguladores; ou (iii) o impacto de indicadores econômicos de mercado sobre as receitas. Diferentemente das OPS de Autogestão, que tem seu público alvo mais cativo, as OPS do tipo Cooperativa e Medicina de Grupo estão mais sensíveis às mudanças no mercado de trabalho e também à rotatividade de beneficiários. Dados da ANS (2015) indicam que, de janeiro a março de 2017, a rotatividade para planos coletivos da Autogestão foi de 1,8\%, para a modalidade Cooperativa a taxa foi de $7,3 \%$, já para a modalidade Medicina de Grupo a mesma taxa foi de 9,4\%.

No caso das operadoras da modalidade Filantropia, a amostra foi composta, na média, por 50 empresas. Ao menos $40 \%$ dessas organizações foram consideradas eficientes pelo modelo, em 4 dos 5 anos analisados. Isso indica que o número de benchmarks de mercado entre as organizações filantrópicas foi relativamente alto, ao ser comparado com as demais modalidades. Essa homogeneidade pode indicar a forte presença da regulação do setor, que faz com que elas adotem uma estrutura parecida, e também ao fato dessas entidades terem a menor taxa de sinistralidade entre as modalidades de operadoras médico-hospitalares.

Adicionalmente, de posse dos escores de eficiência do modelo 2, foram realizados testes de Kruskal-Wallis no intuito de confirmar estatisticamente se as médias dessas populações (modalidades de OPS) diferem. Para verificar a existência de diferenças entre modalidades, analisou-se o modelo 2 (que analisa todas as OPS conjuntamente). O cálculo do modelo 2 foi realizado para validar a existência de diferenças ao analisar as operadoras conjuntamente e separadamente. A importância desse teste reside na validação da 
impossibilidade de realizarem-se análises financeiras sem que haja a devida separação por tipo de organização. Os resultados dos testes encontram-se na Tabela 3.

Tabela 3 - Estatísticas do qui-quadrado para os escores de eficiência ao se considerar a modalidade das OPS, por ano - Modelo 2

\begin{tabular}{l|l|l|l|l|l|l}
\hline & $\mathbf{2 0 1 0}$ & $\mathbf{2 0 1 1}$ & $\mathbf{2 0 1 2}$ & $\mathbf{2 0 1 3}$ & $\mathbf{2 0 1 4}$ & $\mathbf{2 0 1 5}$ \\
\hline Modelo 2 & $31,47 * *$ & $38,84 * *$ & $9,91 *$ & $11,82 * *$ & $48,12 * *$ & $36,34 * *$ \\
\hline
\end{tabular}

Legenda - *Significativo a menos de 5,0\% segundo o teste de Kruskal-Wallis.

${ }^{* *}$ Significativo a menos de 1,0\% segundo o teste de Kruskal-Wallis.

Fonte: Dados da pesquisa.

A partir dos testes de Kruskal-Wallis, foi possível concluir que existem diferenças estatisticamente válidas para os escores de eficiência do modelo 2 quando comparadas entre as modalidades das OPS. Ou seja, existem indícios estatisticamente válidos de que os escores de eficiência de uma modalidade são diferentes de outra. Em todos os anos estudados, observou-se que as organizações da modalidade Autogestão apresentaram escores de eficiência consideravelmente maiores que os demais grupos. Essa eficiência consideravelmente maior pode ser justificada pelos baixos níveis de gastos com despesas administrativas e comerciais.

Para identificar os benchmarks e possíveis melhorias para cada grupo de OPS, fez-se necessário analisar o modelo 1 (que analisa as organizações separadamente). Assim, analisaram-se ainda os outputs quanto à porcentagem de organizações consideradas eficientes pelo modelo 1 que estão com os indicadores no terceiro quartil (quartil superior). Nesse caso, o terceiro quartil destaca $25 \%$ dos melhores resultados dos indicadores analisados. A porcentagem de organizações eficientes e que tem seus indicadores no $3^{\circ}$ quartil (conhecido como quartil superior, por selecionar $25 \%$ dos valores mais elevados da amostra) encontramse na Tabela 4.

Tabela 4 - Porcentagem de organizações eficientes no $3^{\circ}$ quartil, segundo outputs

\begin{tabular}{l|l|l|l|l|l|l}
\hline & $\mathbf{2 0 1 0}$ & $\mathbf{2 0 1 1}$ & $\mathbf{2 0 1 2}$ & $\mathbf{2 0 1 3}$ & $\mathbf{2 0 1 4}$ & $\mathbf{2 0 1 5}$ \\
\hline ROA & $43 \%$ & $41 \%$ & $45 \%$ & $54 \%$ & $48 \%$ & $49 \%$ \\
\hline ROE & $37 \%$ & $39 \%$ & $43 \%$ & $41 \%$ & $42 \%$ & $42 \%$ \\
\hline MEBITDA & $53 \%$ & $64 \%$ & $61 \%$ & $63 \%$ & $65 \%$ & $53 \%$ \\
\hline LG & $34 \%$ & $41 \%$ & $40 \%$ & $41 \%$ & $43 \%$ & $41 \%$ \\
\hline
\end{tabular}

Fonte: Dados da pesquisa.

A partir da Tabela 4, é possível verificar que, dentre os outputs considerados no modelo, aquele que apresenta maior participação de organizações eficientes em seu $3^{\circ}$ quartil 
é o MEBITDA. Em todos os anos analisados, esse é o indicador que apresenta mais de $50 \%$ de organizações consideras eficientes no $3^{\circ}$ quartil. $O$ mesmo não acontece com os indicadores ROA, ROE e LG. Isso pode demonstrar que a estrutura de investimentos tem pouca influência na eficiência da OPS e, por outro lado, que as operações empresariais têm o maior impacto na eficiência. Assim, pode-se concluir que OPS com ativo elevado não necessariamente estão associadas a um bom nível de eficiência.

De modo a discutir as possibilidades de melhoria nos padrões de desempenho das OPS, a partir da identificação dos benchmarks do mercado, foram identificados os alvos médios de eficiência para cada segmento analisado no modelo 1. A análise dos alvos médios mostra a situação atual e a situação-alvo para cada modalidade. A situação-alvo refere-se à necessidade de melhoria possível para que aquelas OPS que não foram consideradas eficientes possam conseguir alcançar a eficiência medida pelo modelo. Assim, essa seria a medida de melhoria necessária para que as organizações não consideradas eficientes alcancem a eficiência de seus benchmarks. A evolução dos alvos médios nos anos analisados neste estudo está disposta nas Tabelas 5, 6, 7 e 8.

Tabela 5 - Evolução dos alvos médios modalidade Autogestão

\begin{tabular}{l|l|l|l|l|l|l}
\hline & $\mathbf{2 0 1 0}$ & $\mathbf{2 0 1 1}$ & $\mathbf{2 0 1 2}$ & $\mathbf{2 0 1 3}$ & $\mathbf{2 0 1 4}$ & $\mathbf{2 0 1 5}$ \\
\hline ENDIV & $-16 \%$ & $-11 \%$ & $-12 \%$ & $-39 \%$ & $-13 \%$ & $-12 \%$ \\
\hline CE & $-17 \%$ & $-11 \%$ & $-7 \%$ & $0 \%$ & $0 \%$ & $0 \%$ \\
\hline DM & $-15 \%$ & $-7 \%$ & $-14 \%$ & $-15 \%$ & $-6 \%$ & $-6 \%$ \\
\hline COMBA & $-4 \%$ & $0 \%$ & $-1 \%$ & $-3 \%$ & $-5 \%$ & $0 \%$ \\
\hline PMCR & $-32 \%$ & $-25 \%$ & $-21 \%$ & $-44 \%$ & $-20 \%$ & $-3 \%$ \\
\hline ROA & $6 \%$ & $10 \%$ & $12 \%$ & $6 \%$ & $8 \%$ & $5 \%$ \\
\hline ROE & $6 \%$ & $2 \%$ & $9 \%$ & $5 \%$ & $3 \%$ & $3 \%$ \\
\hline MEBITDA & $4 \%$ & $5 \%$ & $16 \%$ & $5 \%$ & $7 \%$ & $9 \%$ \\
\hline LG & $11 \%$ & $39 \%$ & $22 \%$ & $30 \%$ & $22 \%$ & $13 \%$ \\
\hline
\end{tabular}

Fonte: Dados da pesquisa.

Tabela 6 - Evolução dos alvos médios modalidade Cooperativa

\begin{tabular}{l|r|r|r|r|r|r}
\hline & $\mathbf{2 0 1 0}$ & $\mathbf{2 0 1 1}$ & $\mathbf{2 0 1 2}$ & $\mathbf{2 0 1 3}$ & $\mathbf{2 0 1 4}$ & $\mathbf{2 0 1 5}$ \\
\hline ENDIV & $-7 \%$ & $-9 \%$ & $-8 \%$ & $-23 \%$ & $-3 \%$ & $-10 \%$ \\
\hline CE & $-9 \%$ & $-3 \%$ & $-6 \%$ & $0 \%$ & $-1 \%$ & $0 \%$ \\
\hline DM & $-14 \%$ & $-8 \%$ & $-9 \%$ & $-9 \%$ & $-6 \%$ & $-9 \%$ \\
\hline COMBA & $0 \%$ & $0 \%$ & $0 \%$ & $-3 \%$ & $-2 \%$ & $0 \%$ \\
\hline PMCR & $-11 \%$ & $-19 \%$ & $-16 \%$ & $-12 \%$ & $-12 \%$ & $-17 \%$ \\
\hline ROA & $3 \%$ & $6 \%$ & $5 \%$ & $3 \%$ & $3 \%$ & $11 \%$ \\
\hline ROE & $1 \%$ & $4 \%$ & $5 \%$ & $3 \%$ & $0 \%$ & $5 \%$ \\
\hline MEBITDA & $1 \%$ & $6 \%$ & $7 \%$ & $2 \%$ & $3 \%$ & $9 \%$ \\
\hline LG & $13 \%$ & $7 \%$ & $12 \%$ & $31 \%$ & $3 \%$ & $18 \%$ \\
\hline
\end{tabular}

Fonte: Dados da pesquisa. 
Tabela 7 - Evolução dos alvos médios modalidade Filantropia

\begin{tabular}{l|r|r|r|r|r|r}
\hline & $\mathbf{2 0 1 0}$ & $\mathbf{2 0 1 1}$ & $\mathbf{2 0 1 2}$ & $\mathbf{2 0 1 3}$ & $\mathbf{2 0 1 4}$ & $\mathbf{2 0 1 5}$ \\
\hline ENDIV & $-13 \%$ & $-2 \%$ & $-3 \%$ & $-18 \%$ & $-6 \%$ & $-9 \%$ \\
\hline CE & $-2 \%$ & $-4 \%$ & $-1 \%$ & $0 \%$ & $0 \%$ & $0 \%$ \\
\hline DM & $-4 \%$ & $-7 \%$ & $-6 \%$ & $-8 \%$ & $-4 \%$ & $-7 \%$ \\
\hline COMBA & $0 \%$ & $0 \%$ & $-1 \%$ & $-10 \%$ & $-15 \%$ & $-1 \%$ \\
\hline PMCR & $-8 \%$ & $-8 \%$ & $-5 \%$ & $-5 \%$ & $-8 \%$ & $-13 \%$ \\
\hline ROA & $4 \%$ & $7 \%$ & $4 \%$ & $3 \%$ & $4 \%$ & $4 \%$ \\
\hline ROE & $3 \%$ & $0 \%$ & $4 \%$ & $5 \%$ & $2 \%$ & $4 \%$ \\
\hline MEBITDA & $1 \%$ & $8 \%$ & $15 \%$ & $5 \%$ & $5 \%$ & $40 \%$ \\
\hline LG & $8 \%$ & $10 \%$ & $14 \%$ & $33 \%$ & $13 \%$ & $10 \%$ \\
\hline
\end{tabular}

Fonte: Dados da pesquisa.

Tabela 8 - Evolução dos alvos médios modalidade Medicina de Grupo

\begin{tabular}{l|r|r|r|r|r|r}
\hline & $\mathbf{2 0 1 0}$ & $\mathbf{2 0 1 1}$ & $\mathbf{2 0 1 2}$ & $\mathbf{2 0 1 3}$ & $\mathbf{2 0 1 4}$ & $\mathbf{2 0 1 5}$ \\
\hline ENDIV & $-9 \%$ & $-14 \%$ & $-35 \%$ & $-15 \%$ & $-13 \%$ & $-7 \%$ \\
\hline CE & $-2 \%$ & $-3 \%$ & $-2 \%$ & $0 \%$ & $0 \%$ & $0 \%$ \\
\hline DM & $-13 \%$ & $-15 \%$ & $-16 \%$ & $-28 \%$ & $-15 \%$ & $-11 \%$ \\
\hline COMBA & $-8 \%$ & $0 \%$ & $0 \%$ & $-4 \%$ & $-10 \%$ & $0 \%$ \\
\hline PMCR & $-22 \%$ & $-24 \%$ & $-51 \%$ & $-7 \%$ & $-13 \%$ & $-14 \%$ \\
\hline ROA & $5 \%$ & $8 \%$ & $11 \%$ & $6 \%$ & $3 \%$ & $4 \%$ \\
\hline ROE & $6 \%$ & $6 \%$ & $11 \%$ & $4 \%$ & $3 \%$ & $3 \%$ \\
\hline MEBITDA & $6 \%$ & $9 \%$ & $15 \%$ & $4 \%$ & $4 \%$ & $5 \%$ \\
\hline LG & $16 \%$ & $27 \%$ & $118 \%$ & $28 \%$ & $17 \%$ & $15 \%$ \\
\hline
\end{tabular}

Fonte: Dados da pesquisa.

A partir das Tabelas 5, 6, 7 e 8, observa-se que, nas modalidades Autogestão, Cooperativa e Medicina de Grupo, ao longo do período analisado, foram identificadas boas possibilidades de melhoria nos indicadores PMCR e LG. Já, na modalidade Filantropia, existem boas possibilidades de melhoria nos indicadores MEBITDA e LG.

É possível ainda observar que os níveis de melhoria para cada modalidade de OPS é diferente. Como exemplo, pode-se citar o indicador PMCR que, está relacionado ao prazo de recebimento de clientes, e apresentou: (i) na modalidade Autogestão, possibilidades de melhoria de 44\% no ano de 2013 (ver tabela 5); (ii) na modalidade Cooperativa, possibilidades de melhoria de 19\% no ano de 2011 (ver tabela 6); (iii) na modalidade Medicina de Grupo, possibilidades de melhoria de 51\% no ano de 2012 (ver Tabela 8). Por outro lado, alguns indicadores se mostraram bem próximos dos níveis eficientes em seus grupos, a saber: CE, COMBA e ROE. Nesses indicadores, as possibilidades de melhoria não passaram de $6 \%$, em média. No que se refere às características institucionais empresariais, conclui-se que, de forma geral, as operadoras da modalidade Autogestão demostraram uma eficiência superior, quando comparada às demais modalidades, e que as entidades do tipo 
Medicina de Grupo apresentam possibilidades de melhoria relevantes, quando comparado com seus pares, quanto aos indicadores LG e DM. Cabe indicar que dados da ANS (2017) afirmam que a modalidade Autogestão tem a menor rotatividade entre as modalidades de OPS, enquanto a modalidade Medicina de Grupo tem a maior rotatividade para planos coletivos e individuais. A maior rotatividade em um plano pode levar ao aumento das despesas médicas, devido à mudança de rede de atendimento e descontinuidade do tratamento médico. A indicação de importância das operações empresariais, concluída a partir da Tabela 4, traz indícios de que a gestão empresarial pode ser fator determinante para a eficiência dessas organizações. Dessa maneira, é importante que o gestor esteja atento a questões operacionais e que ele procure ações preventivas de modo a diminuir seus custos/despesas, a rotatividade de clientes e aumentar a receita empresarial.

De forma análoga, as possibilidades de melhoria desses indicadores também são alteradas conforme a modalidade analisada. É esperado que as diferenças entre os resultados financeiros das modalidades possam ser justificadas (i) pela faixa etária da carteira de clientes; (ii) pela taxa de sinistralidade das modalidades; (iii) pela taxa de rotatividade dos clientes; (iv) pela quantidade de planos coletivos e individuais ofertados; e (v) pela região de atuação, dentre outras

\section{CONCLUSÃO}

Esta pesquisa vislumbrou apresentar e discutir algumas questões relacionadas à gestão financeira de OPS que atuaram no mercado brasileiro de saúde suplementar nos anos de 2010 a 2015. Esta análise se justifica devido às grandes proporções do setor no Brasil e, também, à possibilidade de melhoria na gestão por meio da teoria institucional e da avaliação de desempenho. Buscando analisar a eficiência das OPS, utilizando indicadores financeiros, segregando-as em função da modalidade, realizou-se o cálculo da eficiência a partir de dois modelos propostos. Com o cálculo da eficiência dos dois modelos, foi possível verificar que, conforme sugerido pela teoria institucional, os escores de eficiência se mostraram homogêneos. Ao analisar alguns indicadores financeiros das OPS foram encontradas evidências de que a modalidade tem relação com a eficiência dessas organizações.

De modo a discutir as possibilidades de melhoria nos padrões de desempenho das OPS após a identificação dos benchmarks do mercado, foram identificados, a partir do modelo 1 , os alvos médios de eficiência para cada segmento analisado. De forma geral, conclui-se que os indicadores PMCR, MEBITDA e LG são os que apresentam, em média, maior 
possibilidade de melhoria para atingir os níveis dos benchmarks do mercado. Por outro lado, os indicadores CE, COMBA e ROE mostraram- se os mais próximos dos níveis das organizações consideradas eficientes pelo modelo 1 .

Após o cumprimento dos objetivos específicos, foi possível analisar o mercado de saúde suplementar ao longo do período de forma mais abrangente, de modo a identificar indícios de possíveis isomorfismos organizacionais e analisar o desempenho econômicofinanceiro das OPS que possuem características institucionais diferentes. Assim, tem-se a suspeita de que a modalidade da operadora tem relação direta com a eficiência financeira da organização. Esse é um importante achado desse estudo, uma vez que demonstra que as OPS devem ser comparadas somente com seus pares - mesma modalidade. Encontramos indícios de que as diferenças existentes entre elas impactam os indicadores e a eficiência dessas organizações.

Cabe indicar que o presente trabalho apresenta algumas limitações. Quanto aos testes de Kruskal-Wallis e Correlação de Spearman, é possível acrescentar que ambos são considerados menos assertivo para se analisar os dados, quando comparados aos testes paramétricos, que já apresentam uma distribuição específica determinada. Entretanto, diante da não normalidade dos dados desta pesquisa, optou-se por utilizá-los mesmo com tal limitação. Quanto ao modelo DEA utilizado, é sabido que os resultados não podem ser generalizados, devido ao fato do modelo apresentar a eficiência apenas em referência às OPS analisadas na amostra. Como sugestão de estudos futuros, espera-se que novas pesquisas testem a diferenciação existente entre modalidades e seus possíveis efeitos nos indicadores financeiros.

\section{AGRADECIMENTOS}

O presente trabalho foi realizado com apoio da Coordenação de Aperfeiçoamento de Pessoal de Nível Superior - Brasil (CAPES) - Código de Financiamento 001, do Conselho Nacional de Desenvolvimento Científico e Tecnológico - Brasil (CNPq) e da Fundação de Amparo à Pesquisa do Estado de Minas Gerais - Brasil (FAPEMIG).

\section{REFERÊNCIAS}

AGÊNCIA NACIONAL DE SAÚDE SUPLEMENTAR (ANS). Ministério da Saúde/ANS. Caderno de Informações da Saúde Suplementar, Rio de Janeiro, 2017. 
AGÊNCIA NACIONAL DE SAÚDE SUPLEMENTAR (ANS). Ministério da Saúde/ANS.

Perfil do Setor - Dados Gerais. 2018a. Disponível em: http://www.ans.gov.br/perfil-dosetor/dados-gerais. Acesso em 17 jan. 2019.

AGÊNCIA NACIONAL DE SAÚDE SUPLEMENTAR (ANS). Ministério da Saúde/ANS.Operadoras em regime especial de liquidação e em falência, 2018b.

Disponível em: www.ans.gov.br/planos-de-saude-e-operadoras/informacoes-e-avaliacoes-deoperadoras/operadoras-em-regime-especial-de-liquidacao-ou-falencia. Acesso em: 20 mai. 2019.

ALLEN, D. et al. Towards a sociology of healthcare safety and quality. Sociology of Health \& Illness, v. 38, n. 2, p. 181-197, 2016.

ALTMAN, E. I. Financial ratios, discriminant analysis and the prediction of corporate bankruptcy. The Journal of Finance, v. 23, n. 4, p. 589-609, 1968.

ARAUJO, A. A. da S.; SILVA, J. R. S. Análise de tendência da sinistralidade e impacto na diminuição do número de operadoras de saúde suplementar no Brasil. Ciência \& Saúde Coletiva, Rio de Janeiro, v. 23, n. 8, p. 2763-2770, 2018.

AVELAR, E. A. et al. Efeitos da regulação da Agência Nacional de Saúde Suplementar (ANS) sobre a distribuição de resultados em operadoras de planos de saúde. In XIX USP InternationalConference in Accounting. 2019.

BRAITHWAITE, J. et al. Healthcare reform, quality and safety: perspectives, participants, partnerships and prospects in 30 countries. Cidade: Farnham - Surrey. Ashgate Publishing, 2015.

COOPER, D. R.; SCHINDLER, P. S. Métodos de pesquisa em administração, 7. ed. Porto Alegre: Bookman, 2003.

CRESWELL, J. W. Projeto de pesquisa: métodos qualitativo, quantitativo e misto. 2. ed. Porto Alegre: Artmed, 2007.

DAMODARAN, A. Valuation approaches and metrics: a survey of the theory and evidence. New York: Now Publishers, 2007.

DIMAGGIO, P. J.; POWELL, W. W. The iron cage revisited: institutional isomorphism and collective rationality in organizational fields. American Sociological Review, v. 48, p. 14760, 1983.

GHALAYINI, A. M.; NOBLE, J. S. The changing basis of performance measurement. International Journal of Operations \& Production Management, v. 16, n. 8, p. 63-80, 1996.

GIRDWOOD, S. et al. Primary healthcare delivery models for uninsured low-income earners during the transition to National Health Insurance: perspectives of private South African providers. South African Medical Journal, v. 109, n. 10, p. 771-783, 2019.

GOLANY, B.; ROLL, Y. An application procedure for DEA. Omega, v. 17, n. 3, p. 237-50, 1989. 
HASHIMOTO, P. de A. Análise da eficiência técnica das operadoras de planos de saúde com a utilização da Análise Envoltória de Dados. 2010. 139 f. Dissertação (Mestrado em Ciências Econômicas) - Universidade do Estado do Rio de Janeiro, Rio de Janeiro, 2010.

HENRY, G. T.; MARK, M. M. Beyond use: understanding evaluation's influence on attitudes and actions. American Journal of Evaluation, v. 24, n. 3, p. 293-314, 2003.

HOLLINGSWORTH, B. The measurement of efficiency and productivity of health care delivery. Health Economics, v. 17, n. 10, p. 1107-1128, 2008.

HUNG, H. Normalized collective corruption in a transitional economy: small treasuries in large chinese enterprises. Journal of Business Ethics, v. 79, n. 1-2, p. 69-83, 2008.

KAPLAN, R. S.; NORTON, D. P. The balanced scorecard: measures that drive performance. Harvard Business Review, v. 83, n. 7, p. 172, 2005.

LAKATOS, E. M.; MARCONI, M. de A. Metodologia cientifica: ciência e conhecimento científico. 7. ed. São Paulo: Atlas. 2004.

MACEDO, M. A. da S.; SILVA, F. de F.; SANTOS, R. M. Análise do mercado de seguros no Brasil: uma visão do desempenho organizacional das seguradoras no ano de 2003. Revista Contabilidade Finanças, São Paulo, v. 17, n. 2, p. 88-100, 2006.

MACHADO-DA-SILVA, C. L.; GONÇALVES, S. A. Nota técnica: a teoria institucional. In: CLEGG, S. R.; HARDY, C.; NORD, W. R. (Org. edição original); CALDAS, M.; FACHIN, R.; FISCHER, T. (Org. edição brasileira). Handbook de estudos organizacionais: modelos de análise e novas questões em estudos organizacionais. São Paulo: Atlas, 2006, cap. 7, p. 220- 226.

MALTA, D. C. et al. Perspectivas da regulação na saúde suplementar diante dos modelos assistenciais. Ciência \& Saúde Coletiva, Rio de Janeiro, v. 9, n. 2, p. 433-44, 2004.

MARÔCO, J. Análise estatística com o PASW Statistics (ex-SPSS). Pêro Pinheiro: Report Number, 2010.

MELLO, J. C. C. B. S. de et al. Curso de análise de envoltória de dados. In.: XXXVII SIMPÓSIO BRASILEIRO DE PESQUISA OPERACIONAL. Gramado. Anais... Gramado: Universidade Federal Fluminense, 2005.

MELO, A. J. M.; DANTAS, N. A. S. A sustentabilidade econômico-financeira das operadoras de planos de saúde diante da concessão indiscriminada de tutelas de urgência no Brasil.

Revista Brasileira de Políticas Públicas, Brasília, v. 8, n. 3, p. 411-433, 2018.

NISHIJIMA, M.; BIASOTO JUNIOR, G. O padrão de financiamento da saúde nos países da América. In. BIASOTO JUNIOR, G.; SILVA, P. L. de B.; DAIN, S. (Orgs.). Regulação do setor saúde nas Américas: as relações entre o público e o privado numa abordagem sistêmica. Brasília: Organização Pan-Americana da Saúde, 2006.

PEREIRA, R. C.; SILVESTRE, R. M. Regulação e modelos assistenciais em saúde suplementar: produção científica da rede de centros colaboradores da ANS-2006/2008. Brasília: Organização Pan-Americana da Saúde, 2009. Série Técnica Projeto de Desenvolvimento de Sistemas e Serviços de Saúde. 
PIRES, J. M.; TORRES, A. S. de V.; ALMEIDA, V. M. C. de. Unimed Leste Fluminense: alinhando os canais para crescer. REAd. Revista Eletrônica Administração, Porto Alegre, v. 19, n. 3, p. 796-819, 2013.

SAMPAIO, L. M. D.; OLIVEIRA, M. J. F. de; IGNACIO, A. A. V. Análise e classificação das operadoras da saúde suplementar. XLI SBPO 2009 - Pesquisa Operacional na Gestão do Conhecimento, p. 1365, Porto Seguro, Bahia, Brasil.

SAMPIERI, R. H.; COLLADO, C. F.; LUCIO, P. B. Metodologia de pesquisa. 3. ed. São

Paulo: MacGraw-Hill, 2006.

SOUZA, A. A. de. et al. Controle de gestão em organizações hospitalares. REGE Revista de Gestão, São Paulo, v. 16, n. 3, p. 15-29, 2009.

STEVENSON, W. J. Estatística aplicada à administração. 1. ed. São Paulo: Harbra, 2001.

VIACAVA, F. et al. Avaliação de desempenho de sistemas de saúde: um modelo de análise.Ciência \& Saúde Coletiva, Rio de Janeiro, v. 17, n. 4, p. 921-934, 2012.

ZSIDÓ, K. E. et al. Application of traditional and new approach methods in business performance measurement. Cross Cultural Management Journal, n. 7, p. 51-7, 2015.

\section{DADOS DOS AUTORES}

Nome: Daniele Oliveira Xavier

E-mail: danieleoliveiraxavier@gmail.com

Currículo Lattes: http://lattes.cnpq.br/5701030096056503

Doutoranda e Mestre em Administração/Finanças pela Universidade de Minas Gerais; Especialista em Gestão Púbica pela Universidade do Estado de Minas Gerais; Bacharel em Ciências Contábeis pela Universidade Federal de Minas Gerais. Atualmente, é gestora em Ciência e Tecnologia - Contabilidade na Fundação João Pinheiro. Tem experiência na área de Ciências Contábeis, Administração e Educação à Distância.

Nome: Antônio Artur de Souza

E-mail: antonioarturdesouza@gmail.com

Currículo Lattes: http://lattes.cnpq.br/0597505816212353

Doutor em Management Science pela Universidade de Lancaster; pós-doutor em Finanças pela Universidade de Grenoble; Mestre em Engenharia de Produção pela Universidade Federal de Santa Catarina; Especialista em Educação à Distância pela Universidade Federal Fluminense; Bacharel em Ciências Contábeis pela Universidade Federal de Santa Catarina. Atualmente é professora da Universidade Federal de Minas Gerais. Tem experiência nas áreas de Administração e Ciências Contábeis, nas especialidades de Finanças, Orçamento Empresarial, Contabilidade Financeira, Planejamento Tributário, Contabilidade Gerencial e Análise e Gestão de Custos. 